\title{
RED VARIABLES OF THE HALO AND THE \\ GLOBULAR CLUSTERS
}

\author{
T. LLOYD EVANS \\ Radcliffe Observatory, South African Astronomical Observatory, South Africa
}

\begin{abstract}
The present state of knowledge of the red variables in the halo and globular clusters is summarized. Techniques for their discovery are described. The correlations between their properties and those of the globular clusters to which they belong are discussed. The evolutionary state of the variables is considered and stars are assigned tentatively to the first or second ascent of the giant branch or to the excursions from the asymptotic giant branch resulting from instabilities in the He-burning shell.

The assumption that a recognizable type of variable represents a unique type of star leads to the conclusions that the bulk of the stars in the central bulge of the Galaxy are more metal-rich than 47 Tuc and that the Large Magellanic Cloud contains an old, moderately metal-deficient population similar to that represented by clusters such as 47 Tuc in the Galaxy while the Small Magellanic Cloud does not.
\end{abstract}

\section{Introduction}

Detailed understanding of the red variables has been slow to come, in large measure because of the heterogeneity of the field star population and the difficulty in determining such fundamental quantities as mass, luminosity, surface temperature and chemical composition. The briefness of this stage of evolution means that they are not common in star clusters, but the richness of the globular clusters provides us with a useful sample of red variables of the low mass, metal-deficient population. Mass, luminosity and chemical composition may be estimated from the more normal stars in the cluster.

The red variables are generally the coolest stars on the giant branch and so occur at a stage where several points of theoretical interest are found: the helium flash which terminates the first ascent of the giant branch, the thermal instabilities which interrupt the progression up the asymptotic giant branch in the case of a double shell source star, and mass loss which may occur as the climax of the second ascent of the giant branch.

Stars such as these, which are bright, readily identifiable from their variability and at an extreme stage of evolution whose properties may depend sensitively on initial conditions, are especially valuable as markers of a particular population in distant stellar systems. An essential preliminary is to determine the correlation between their properties and the parameters which characterize the parent stellar system, and to establish its uniqueness.

\section{Discovery of Red Variables}

Blue sensitive plates have been used almost exclusively in searches for variable stars in globular clusters, which have usually been directed towards finding RR Lyrae stars. Kukarkin (1973) has deduced from Russev's (1971) observations of red variables in 
the moderately metal-poor cluster M3 that $B$ is preferable to $V$ in that the amplitude is larger. It was found in the blink search of several metal-rich clusters (Lloyd Evans and Menzies, 1973) that the $B$ waveband may be superior in an open cluster, where there is a larger choice of comparison stars, but in a crowded cluster the red variables stand out better on a $V$ plate; in extreme cases such as NGC 6388 infrared plates permit the detection of Mira variables. The availability of $V$ and $I$ plate pairs allows a clear distinction between red variables and other stars in a metal-rich cluster.

The optimum spacing of observations for the detection and study of red variables is about $\mathbf{1 0 0}$ times that appropriate to RR Lyrae stars.

There are undoubtedly many more variables of small amplitude still to be discovered (Walker, 1955; Brooke, 1969; Russev, 1971; Eggen, 1972; Lloyd Evans and Menzies, 1973).

\section{Red Variables in Globular Clusters}

There are at present about 140 red variables known in globular clusters; some of these are certainly field stars. The metal-rich clusters are better represented at present, largely because of a search specifically directed towards red variables (Lloyd Evans and Menzies, 1973). It is convenient to discuss the clusters in 3 groups: metal-poor, metal-rich and the apparently unique cluster $\omega$ Cen. The boundary between the metal-poor and metal-rich clusters has been set so that the former do not contain $M$ stars, except that some of the variables may exhibit TiO bands when faint. This information is not contained in conventional colour magnitude diagrams, so we adopt $Q=-0.33$ (van den Bergh, 1967) with the additional constraint, suggested by Joy's (1949) spectra of red variables in globular clusters, that Dickens' (1972) horizontal branch type is $<5$ for the metal-poor clusters*.

\subsection{THE METAL-POOR CLUSTERS}

The amplitudes of the red variables presently known (Hogg, 1973) lie between 0.3 and 1.5 , with a very few having larger amplitude. Walker (1955) has discovered variables of smaller amplitude in M92 and M3; the variables have $M_{V}$ brighter than -2.3 (assuming the RR Lyrae stars have $M_{V}=+0.5$ ) and the amplitude is greater the brighter the star. Russev (1971) has obtained similar results for M3.

Photographic colour magnitude diagrams of 8 clusters show their 10 red variables to lie at or slightly down the red giant branch from the tip. A few lie above the giant branch defined by non-variable stars but by amounts which are scarcely significant in view of the generally poor coverage of the light cycles.

Eggen (1972) has published $B V R I$ photometry of 7 variables in 4 clusters. He finds a pronounced tendency for these stars to lie above the giant branch in the $V, B-V$ and especially the $M_{\text {bol }}, R-I$ diagrams. This is not in complete agreement with the

* Dickens' (1972) horizontal branch types ( $1 \leqq H B \leqq 7)$, ranging from many stars to blueward of the RR Lyr instability strip for 1 to few or no stars to blueward and many to redward for 7, correspond roughly with metallicity: $1 \leqq H B<5$ for metal-poor globular clusters and $5 \leqq H B \leqq 7$ for metal-rich. 
results from photographic photometry of the same clusters. The point is potentially important in the case of NGC 6656, where V5 and V8 were regarded as SRd variables by Feast (1973a) on the basis of Joy's (1949) discovery of emission lines in their spectra. These two stars were considered by Lloyd Evans and Menzies (1973) as analogous to stars in the metal-rich globular clusters (such as R10 in $47 \mathrm{Tuc}$ ) which lie above and to the blue of the other red variables on the giant branch.

The two types of star are quite distinct in the metal-rich clusters, where the Mira variables, of which the SRd variables are thought to be the metal-poor analogues, describe long tracks beyond the tip of the giant branch (Lloyd Evans, 1974a). The emission line star V95 in NGC 5272, regarded as an SRd variable by Feast (1973a), does indeed lie at the tip of the giant branch (Arp, 1955).

A new photographic study of NGC 6656 on the BVI system (Lloyd Evans, in preparation) shows that on one night the three red variables V5, V8 and V9 fell at or slightly below the tip of the giant branch. This can only be reconciled with Eggen's (1972) observations of V5 and V8 if both stars were at minimum light and the amplitudes are much greater than those quoted in the past. The problem deserves further study.

The majority of the red variables in metal-poor clusters do evidently lie on the giant branch defined by non-variable stars. Their evolutionary state is probably similar to that of the non-TiO variables in $\omega$ Cen (see Section 3.3).

Periods are available for 19 stars in 12 clusters. The range is $\mathbf{4 0}$ to 131 days, with a mean of 88 days. There is little correlation between amplitude and period.

\subsection{THE METAL-RICH CLUSTERS}

An important study of the 8 known red variables in 47 Tuc was published by Arp et al. (1963). A survey of red variables in metal-rich clusters by Lloyd Evans and Menzies (1973 and in preparation), also Lloyd Evans (1974a) and less extensive work by others, reveals a total of 23 certain and several probable red variables in 47 Tuc. The main properties are:

(1) These stars nearly all lie on the giant branch defined by non-variables, though some of the larger amplitude stars cannot be placed in the colour magnitude diagram with precision (Lloyd Evans, 1974a).

(2) Variability sets in rather abruptly between $(V-I)_{\mathrm{K}}=1.7$ and 1.8, apparently without the slow increase of amplitude with increasing luminosity shown in M3 and M92* (Lloyd Evans and Menzies, 1973; Lloyd Evans, 1974a).

(3) The variables are all $M$ stars, except that $\mathrm{R} 10$ does not show $\mathrm{TiO}$ at all times (Feast and Thackeray, 1960; Lloyd Evans, 1974a).

(4) The stars mainly fall in three period ranges: 3 Miras with $P=192-212$ days, 2 semiregulars with $\Delta m \sim 1^{m}$. 5 and $P \sim 160$ days, and 3 'irregulars' with ill-defined cycles of $\sim 50$ days and $\Delta m \sim 0.7$ (Arp et al., 1963). Eggen (1973a) has reported periods of

* The form of the giant branch in metal-rich clusters requires the use of a temperature criterion rather than absolute magnitude. $B-V$ is useless because TiO blanketing cancels the increase in $B-V$ at low temperatures, but TiO increases the temperature sensitivity of $V-I$. 
100 days for W12 and 70 days for the possible outlying member, Leiden Variable 60. Periods and accurate photometry are required for the new variables. The existing data do not provide sufficiently accurate mean colours to determine whether there is a clumping in colour analogous to that in period and amplitude.

(5) The limited data on bolometric magnitudes (Eggen, 1972; Glass and Feast, 1973) suggest that most of the variables could be on their first ascent of the giant branch. The Mira variables may be brighter than the theoretical helium flash and so presumably on a second ascent of the giant branch (Lloyd Evans, 1974a).

(6) R10 is significantly brighter than the giant branch at its value of $\left(V-I_{K}\right)$, and is bluer than the other variables. The TiO bands are sometimes absent (Lloyd Evans, 1974a) and the $\mathrm{H}$ lines are weak at times, suggesting incipient emission (Feast and Thackeray, 1960). It is one of several deviant stars, all probable members, which may be undergoing excursions from the giant branch as a result of helium shell flashes. (Lloyd Evans, 1974a).

Lloyd Evans and Menzies (1973, and in preparation) have discussed observations of 54 variables (some probably non-members) in 9 other clusters. The situation is qualitatively similar in that most of the variables lie on or near the giant branch. The height of the giant branch above the horizontal branch is less and the mean colour of the variables is redder in the more metal-rich clusters. Glass and Feast (1973) find the same relation between temperature and $\mathrm{TiO}$ band strength (which largely determines $(V-I)$ colour) for stars of different metal content, so the onset of variability must occur at lower temperature for the more metal-rich stars.

The Mira variables of known period now number 8: 6 stars in 3 clusters fall in the narrow period range 190-212 days, despite a range in form of the colour magnitude diagram of the parent clusters, but those in the metal-rich clusters NGC 5927 and NGC 6553 have $P=312^{\mathrm{d}}$ and $P=265^{\mathrm{d}}$ respectively (Lloyd Evans and Menzies, 1973, and in preparation; Andrews et al., 1974). This suggests that the period is longer the greater the metal content.

Reliable periods are not available yet for the variables of smaller amplitude in these clusters.

The evolutionary states of the red variables may be summarized as follows:

The bulk of the smaller amplitude variables fall on the Hayashi line and may be on their first ascent of the giant branch, while the Mira variables may fall on an extension of the Hayashi line but are perhaps too luminous to be pre-He flash stars. The few stars above the giant branch and bluer than the other variables may be undergoing an excursion from the giant branch as a result of instabilities in the He-burning shell.

Improved theoretical models are required, with particular reference to the envelope models and to denser coverage of the higher range of chemical composition.

\section{3. $\omega$ Centauri}

$\omega$ Cen, the richest accessible globular cluster, is a metal-poor cluster whose red variables fall in two distinct groups. One group consists of stars without TiO bands: these 
cluster around the tip of the giant branch like those in other metal-poor clusters and may comprise all stars with $(B-V)_{0}>1.47$ (Dickens and Woolley, 1967; Cannon and Stobie, 1973). They appear to scatter both above and below the mean giant branch (Eggen, 1972; Dickens et al., 1972; Wing, 1973); the non-variables also define a much broader giant branch than that of other clusters (Cannon and Stobie, 1973).

Glass and Feast (1973) find that this giant tip is in satisfactory agreement with theoretical calculations of the location of the helium flash for appropriate chemical composition.

The periods are available for 7 of these stars and range from 33 to 124 days. The amplitudes range from $0^{m} \cdot 3$ to $1^{m} \cdot 1$, being smaller for the stars of shortest period. The position of these stars in the colour magnitude diagram (Dickens et al., 1972) appears to be correlated with period, the stars of short period being fainter.

The second group comprises 4 stars with strong TiO bands. These are RGO 320 (M2), V17 (M3-4, $P \sim 60$ days), V6 (M4-5e, $P \sim 110$ days) and the Mira-like variable V42 ( $P=149$ days) which has type M2e near maximum. These stars have TiO bands at all times (except perhaps for V42 when at maximum) and are quite unlike stars such as V95 in M3 which penetrate the M star region when faint. There are also at least four apparently non-variable cluster members with weaker TiO bands (Lloyd Evans, unpublished); these fall in the gap between the small amplitude variable RGO 320 and the non-TiO stars (cf. Figure 2 of Feast, 1973a).

Photometry in the infrared to $3.5 \mu$ (Glass and Feast, 1973) shows that the M type variables have the same colours as normal $M$ stars of the same spectral type, except for an ultraviolet excess in the former. They have a luminosity similar to that of the red giant tip but are up to $700^{\circ}$ cooler, so that they must lie to the right of the Hayashi line for non-TiO stars unless there is considerable far-infrared emission - perhaps from a cool circumstellar shell which does not redden the star appreciably.

Glass and Feast (1973) suggested that these stars lie on the asymptotic giant branch between the end of the first giant branch and the point where an envelope is ejected. This does not explain why they lie below the Hayashi line, nor why the intermediate stars are non-variable.

Feast (1973b) suggested instead that they may be in a brief evolutionary stage connected with the He-flash at the end of their first ascent of the giant branch.

Both possibilities assume that the $\mathbf{M}$ stars are in a normal evolutionary stage which would be expected to occur in other metal poor clusters. No similar case is yet known, though Hyland and Rodgers (1973) have suggested that V1 and V2 which lie far below the giant branch of NGC 6397 in visible light are cluster members cooler and brighter than the conventional tip of the giant branch.

An alternative explanation is that $\omega$ Cen contains stars of a range of chemical composition or possibly age, as is suggested by the width of the giant branch (assumed real) and other peculiarities. The TiO stars then represent the red giant tip of a group having different properties from the bulk of the stars. The general appearance of these stars - non-variables, variables of increasing amplitude and finally a Mira variable suggests an analogy with the situation found in the metal rich clusters, though the 
combination of a 149-day period Mira with M2-M4 variables of small amplitude violates the usual correlation.

Indications of an inhomogeneous population in $\omega$ Cen should be checked and more metal-poor clusters searched for $\mathbf{M}$ type stars.

\section{Red Variables of the Galactic Halo}

Stars of the halo population may be distinguished by their great distance from the galactic plane or, in the case of stars in the solar neighbourhood, by their high space velocities. Some of these stars are sufficiently unlike stars of other populations to be recognizable without consideration of their kinematics or location.

\subsection{Mira VARIABLES}

An analysis of the velocities of the Me variables was carried out by Feast (1963) and repeated with some additional stars by Feast et al. (1972). The systematic motion of those with periods between 150 and 200 days was comparable to that of such Population II groups as the RR Lyrae variables with $P \sim 0.45$ days. The systematic motions declined rapidly with increasing period, though a number of stars with periods between 200 and 300 days have residual velocities of $100-200 \mathrm{~km} \mathrm{~s}^{-1}$. The stars with $P>300$ days must be predominantly old disk population and those with $P>450$ days may be of age similar to the Hyades, though there are a few stars with $P>400$ days and residual velocities of about $100 \mathrm{~km} \mathrm{~s}^{-1}$. The general trend for the period range up to $\sim 300$ days agrees with the results found for the metal rich globular clusters.

An examination of the data contained in the General Catalogue of Variable Stars (3rd edition) reveals 17 Miras of known period with $|b|>50^{\circ}$ and located more than $1 \mathrm{kpc}$ from the galactic plane, using the absolute magnitude calibration of Clayton and Feast (1970). The period distribution is, for 50 day period intervals about the period shown,

$\begin{array}{cccccccc}125 & 175 & 225 & 275 & 325 & 375 & 400 & \text { days } \\ 2 & 1 & 3 & 5 & 3 & 0 & 3 & \end{array}$

Two stars, AL Boo ( $P=168$ days) and AB Com $(P=201$ days $)$ are at $\sim 10 \mathrm{kpc}$ from the galactic plane and are thus true halo stars. Spectra would be of interest. The long period stars are well represented despite severe observational selection in favour of the brighter stars of shorter period and a survey using infrared plates would be valuable.

\subsection{Semiregular VARiables}

The kinematics of the semiregular variables of type $M$ have been studied by Feast et al. (1972). Most of these stars appear to belong to the old disk population, but those with emission lines in the period range 60-140 days have a considerably higher rms residual velocity. A few have large radial velocities and one of these, CZ Del, was among several stars considered by Eggen (1973b) to be halo stars. CZ Del may be 
similar to V6 in $\omega$ Cen. Glass and Feast (1973) picked out several stars which could be similar to the TiO variables in $\omega$ Cen on the basis of their ultra violet excess.

\subsection{Small amplitude Variables}

Eggen (1970) studied a large sample of red stars, including many variables of small amplitude, in a field centred on the South Galactic Pole. These were divided into young disk, old disk and halo groups on the basis of space motions derived from proper motions and photometric distances. Radial velocities obtained by Jones (1972) and Crampton and Lloyd Evans (1973) suggested that there were few if any halo stars in the sample, most being of the old disk population. A few Lb variables at high northern latitudes likewise appear to be old disk stars (Lloyd Evans, 1974b). Eggen (1973c) has assigned several stars to the Arcturus and $\sigma$ Puppis groups. These have metal abundances of $30 \%$ and $20 \%$ of the solar metal abundance, respectively, and may be comparable with 47 Tuc.

The rarity of halo red variables near the Sun has been demonstrated by the photometry of Eggen (1970) and Stokes (1971), who find a significant proportion of variables only for $\left(V-I_{K}\right) \gtrsim+2.0$, which is redder than the corresponding figure for 47 Tuc despite their use of the accurate photoelectric method which detects very small variations.

A few variables of small amplitude and classed as SRd, such as TY Vir (Preston \& Wallerstein, 1963; Eggen, 1972), are almost certainly identical to the small amplitude variables in halo clusters.

\subsection{THE SRd VARIABLES}

The SRd variables are variable supergiants and giants of spectral classes $F, G, K$. These are mainly weak-lined stars, the temperature being much lower than indicated by the spectral type. There are about 30 stars known, after removing a few hotter stars such as $89 \mathrm{Her}$; almost all are in the Northern hemisphere as a result of more intensive spectroscopic surveys. Periods range from 30 to 237 days and amplitudes from 0.3 to 3.9 mag.; the stars with amplitude $\gtrsim 1^{m}$ occur mainly in the period range 80-135 days.

The mean period for 14 stars with $\mathrm{H}$ emission at times is 102 days, that for 14 stars with TiO absorption at minimum is 110 days and that for 9 stars with amplitudes $\leqslant 1^{m}$ and neither $\mathrm{H}$ emission nor TiO absorption is 73 days.

This is very much what one might expect for the red variables of the metal poor globular clusters, given severe selection by amplitude. Low metal abundances have been found for SX Her and TY Vir (Preston and Wallerstein, 1963).

Joy (1952) found large radial velocities for many of these stars. Feast (1965) showed that the velocities of field SRd variables and the absolute magnitude of those in clusters are consistent with their being a short period, halo continuation of the Mira variables. The weakness of $\mathrm{H} \varepsilon$ emission caused by overlying $\mathrm{Ca}$ II $\mathrm{H}$ absorption was noted as typical of Mira variables.

The considerations above on correlations between period, spectrum and amplitude suggest that the longer period stars having $\mathrm{H}$ emission and often also $\mathrm{TiO}$ absorption 
are analogues of Mira variables, while the short period stars of small amplitude are comparable to the variables of smaller amplitude in the metal rich clusters.

Stars of this type have not yet been studied at great distances from the galactic plane.

\section{The Central Bulge of the Galaxy}

Many periods were found by Gaposchkin (1955) for red variables in the field centred on NGC $6522(l=0: 9, b=-3: 9)$. Some of these were found to be $M$ stars (Nassau and Blanco, 1958). This original survey used blue plates; a new search employed $V$ and $I$ plates, resulting in the discovery of many additional red variables (Lloyd Evans, to be published). These include a substantial number of long period Miras, which were missed earlier because of their faintness. The 180-220 day Miras account for only 6 of 39 Miras with known periods, all but two of the remainder having longer periods. The 47 Tuc population is thus a minor component of the stellar content of this field. The variables of smaller amplitudes are very red, in accordance with this.

This confirms the conclusion of Arp (1965) and Van den Bergh (1971), that the predominant population in this field is not similar to that of the globular clusters. Extrapolation of the trends found in metal-rich globular clusters suggests that the population of the central bulge is metal-rich, in accordance with current thinking. The considerations of Section 4.1 leave open the possibility that the Mira population differs from that of the clusters in age also.

\section{The Magellanic Clouds}

Two fields each in the LMC and SMC are being searched for red variables (Lloyd Evans, 1971). The SMC fields have revealed no stars which can be identified with any of the globular cluster variables; the Miras of 200 day period would have been discovered readily if they were present. The globular clusters in the SMC are unlike those in the Galaxy in containing carbon stars near the red giant tip, suggesting that they are younger than galactic globular clusters (Feast and Lloyd Evans, 1973). The general field, which appears to be similar in stellar content to such clusters as NGC 419, contains large numbers of very red semiregular variables with $V \sim 17-18$ (Lloyd Evans, in preparation).

One field in the LMC contains Mira variables of a wide range of period, including five in the 180-220 day interval. Butler (1971) has surveyed a wider field to brighter limiting magnitude and finds red variables with $P \sim 160$ days and $V_{\max }$ between 15 and 16. These are probably identifiable with the 150-200 day Miras in galactic globular clusters. It is concluded that the LMC, unlike the SMC, does have a substantial component of population similar to that represented by stars of moderate metal deficiency in the Galaxy.

\section{References}

Andrews, P. J., Feast, M. W., Lloyd Evans, T., Menzies, J. W., and Thackeray, A. D. 1974, Observatory 94, 133. 
Arp, H. 1955, Astron J. 60, 317.

Arp, H. 1965, Astrophys. J. 141, 43.

Arp, H., Brueckel, F., and Lourens, J. V. B.: 1963, Astrophys. J. 137, 228.

Bergh, S. van den: 1967, Astron. J. 72, 70.

Bergh, S. van den: 1971, Astron. J. 76, 1082.

Brooke, A. L. 1969, Thesis, Australian National University.

Butler, C. J. 1971, Veröff. Remeis-Sternw. Bamberg 9, Nr. 100, 90.

Cannon, R. D. and Stobie, R. S. 1973, Monthly Notices Roy. Astron. Soc. 162, 207.

Clayton, M. L. and Feast, M. W.: 1970, Monthly Notices Roy. Astron. Soc. 146, 411.

Crampton, D. and Lloyd Evans, T.: 1973, Monthly Notices Roy. Astron. Soc. 162, 11.

Dickens, R. J.: 1972, Monthly Notices Roy. Astron. Soc. 157, 281.

Dickens, R. J., Feast, M. W., and Lloyd Evans, T.: 1972, Monthly Notices Roy. Astron. Soc. 159, 337.

Dickens, R. J. and Woolley, R.: 1967, Roy. Obs. Bull. 128.

Eggen, O. J.: 1970, Astrophys. J. Suppl. 22, 289.

Eggen, O. J.: 1972, Astrophys. J. 172, 639.

Eggen, O. J.: 1973a, Quart J. Roy. Astron. Soc. 14, 433.

Eggen, O. J.: 1973b, Mem. Roy. Astron. Soc. 77, 159.

Eggen, O. J.: 1973c, Astrophys. J. 180, 857.

Feast, M. W.: 1963, Monthly Notices Roy. Astron. Soc. 125, 367.

Feast, M. W.: 1965, Observatory 85, 16.

Feast, M.W.: 1973a, in J. D. Fernie (ed.), Variable Stars in Globular Clusters and in Related Systems, D. Reidel, Dordrecht, p. 131.

Feast, M. W. 1973b, in P. Ledoux, A. Noels, and A. W. Rodgers (eds.), 'Stellar Instability and Evolution' IAU Symp. 59, 93.

Feast, M. W. and Lloyd Evans, T.: 1973, Monthly Notices Roy. Astron. Soc. 164, 15 P.

Feast, M. W. and Thackeray, A. D.: 1960, Monthly Notices.Roy. Astron. Soc. 120, 463.

Feast, M. W., Woolley, R., and Yilmaz, N.: 1972, Monthly Notices Roy. Astron. Soc. 158, 23.

Gaposchkin, S.: 1955, Perem. Zvezdy 10, 337.

Glass, I. S. and Feast, M. W.: 1973, Monthly Notices Roy. Astron. Soc. 163, 245.

Hogg, H. S.: 1973, Publ. David Dunlap Obs. 3, No. 6.

Hyland, A. R. and Rodgers, A. W.: 1973, Verbal report at IAU Symp. 59, 'Stellar Instability and Evolution'.

Jones, D. H. P.: 1972, Astrophys. J. 178, 467.

Joy, A. H.: 1949, Astrophys. J. 110, 105.

Joy, A. H.: 1952, Astrophys. J. 115, 25.

Kukarkin, B. V.: 1973, in J. D. Fernie (ed.), Variable Stars in Globular Clusters and in Related Systems, D. Reidel, Dordrecht, p. 8.

Lloyd Evans, T.: 1971, in A. B. Muller (ed.), The Magellanic Clouds, D. Reidel, Dordrecht, p. 74.

Lloyd Evans, T.: 1974a, Monthly Notices Roy. Astron. Soc. 167, 393.

Lloyd Evans; T.: 1974b, Observatory 94, 179.

Lloyd Evans, T. and Menzies, J. W.: 1973, in J. D. Fernie (ed.), Variable Stars in Globular Clusters and in Related Systems, D. Reidel, Dordrecht, p. 151.

Nassau, J. J. and Blanco, V. M.: 1958, Astrophys. J. 128, 46.

Preston, G. and Wallerstein, G.: 1963, Astrophys. J. 138, 821.

Russev, R. M.: 1971, Perem. Zvezdy 18, 171.

Stokes, N. R.: 1971, Monthly Notices Roy. Astron. Soc. 152, 165.

Walker, M. F.: 1955, Astron. J. 60, 197.

Wing, R. F. 1973, in J. D. Fernie (ed.), Variable Stars in Globular Clusters and in Related Systems, D. Reidel, Dordrecht, p. 164.

\section{DISCUSSION}

D. S. Evans: (1) Are the designations of stars in $\omega$ Centauri from the Herstmonceux proper motion work?

(2) Could you comment on the difference in magnitude of the stars in NGC 6522 and 6528 . Does this not indicate some range in distance?

T. Lloyd Evans: (1) Yes. 
(2) It is likely that NGC 6528 is more metal-rich than NGC 6522 so that the magnitude difference would occur even at equal distance.

$M$. Breger: How would the instability strip in $I,(V-I)$ change if you did not fit the horizontal branch assuming constant absolute magnitudes? A possibility might be the transition periods of $R R$ Lyrae stars, where available.

T. Lloyd Evans: There are insufficient RR Lyrae stars in metal-rich clusters to use the transition period method. Extrapolation of the calculation that the RR Lyraes and hence horizontal branch stars are fainter in more metal-rich cluster would steepen the boundary of the instability strip.

D. H. P. Jones:

(1) Are you sure that $\omega$ Cen is metal deficient? My own observations of the RR Lyraes and the analysis by Dickens and Powell of Fehrenbach's star indicate an underabundance by only a factor of twenty.

(2) Do your observations indicate any stars on the cool side of the Hayashi track?

T. Lloyd Evans:

(1) It is metal deficient by comparison with 47 Tuc which is believed to have an underabundance of metals by only a factor of two. It disobeys the relationships which apply to clusters within the metalrich group.

(2) Feast suggested that stars to the red of the giant branch, or red giant tip, in $\omega$ Cen are to the right of the Hayashi track. However, if we accept a spread of chemical composition within $\omega$ Cen, there will not be a unique Hayashi track in the observational colour magnitude diagram, so such stars may not be to the right of the appropriate Hayashi track. 